\title{
ON ORTHOGONALLY DECOMPOSABLE ORDERED BANACH SPACES
}

\section{SADAYUKI YAMAMURO}

\begin{abstract}
In a Banach lattice or the hermitian part of a $C^{*}$-algebra, every element $a$ admits a decomposition $a=a_{+}-a_{-}$such that $a_{ \pm} \geq 0, N(a)=\left\|a_{+}\right\|$and $N(-a)=\left\|a_{-}\right\|$, where $N$ is the canonical half-norm of the positive cones. In general ordered Banach spaces, this property is related to the order structure of the duality map and the metric projectability of the positive cones, and it turns out to be equivalent to an "orthogonal" decomposability.
\end{abstract}

Throughout this note, we shall denote by $B$ a real Banach space ordered by a closed and proper cone $B_{+}$. Then the dual $B^{*}$ is ordered by the dual cone $B_{+}^{*}=\left\{f \in B^{*}:(f, x) \geq 0\right.$ for all $\left.x \in B_{+}\right\}$.

The canonical half-norm $N$ on $B$, introduced by [2], and in a more general form by [5], is defined by

$$
N(a)=\inf \left\{\|a+x\|: x \in B_{+}\right\} .
$$

Then it is easy to see that $a \in B_{+}$if and only if $N(-a)=0$. Furthermore, $N$ is continuous, $N(a+b) \leq N(a)+N(b), N(\lambda a)=\lambda N(a)$ for $\lambda \geq 0$, and $N(a)+N(-a)=0$ implies $a=0$. It has been proved in [12] that $f \in B^{*}$ is positive, that is, $f \in B_{+}^{*}$, if and only if $f(a) \leq\|f\| N(a)$ for all $a \in B$.

Received 26 April 1984.

Copyright Clearance Centre, Inc. Serial-fee code: 0004-9727/84 $\$ 2.00+0.00$. 
When $B$ is a Banach lattice or the hermitian part of a $C^{*}$-algebra, every element $a \in B$ determines a pair $\left(a_{+}, a_{-}\right)$, the positive and negative parts of $a$, such that $a=a_{+}-a_{-}$. In these two cases we have $N(a)=\left\|a_{+}\right\|$and $N(-a)=\left\|a_{-}\right\|$.

Generally, an ordered Banach space $B$ is said to be $N$-decomposable if every element $a$ of $B$ is expressed as $a=b-c$ such that $b \in B_{+}$, $c \in B_{+}, N(a)=\|b\|$ and $N(-a)=\|c\|$. When the pair $(b, c)$ satisfies only $N(a)=\|b\|$, then $B$ is called $N_{+}$-decomposable. These two notions have been considered in [11] and [13].

When $B$ is a Hilbert space, $B$ is uniquely $N$-decomposable if and only if the positive cone $B_{+}$is self-dual and, in this case, the $N$-decomposition $a=b-c$ is, in fact, an orthogonal decomposition, that is, $(b, c)=0$. The aim of this note is to consider this phenomenon in general ordered Banach spaces.

We shall start with a study on those properties which correspond to the self-duality of the positive cone in Hilbert spaces. Naturally, these properties are expressed in terms of the duality map $F$ of $B$. The basic rôle is played here by another map $H$, which is defined by the canonical half-norm. A relation between $F$ and $H$ is

$$
H(a)=F(b) \cap B_{+}^{*} \cap(b-a)^{0}
$$

if $b \geq a$ and $N(a)=\|b\|$, where

$$
(b-a)^{0}=\left\{f \in B^{*}: f(b-a)=0\right\} .
$$

(See (1.7).) The condition which appears in this statment, that for every $a \in B$ there exists $b \in B$ such that $b \geq a$ and $N(a)=\|b\|$, turns out to be equivalent to the condition that every element of $B$ can be metrically projected to $B_{+}$. This projectability leads us to a notion of orthogonality in $B$, and it will be shown that $B$ is $N$-decomposable if and only if it is orthogonally decomposable in this sense. It then becomes possible to give a simple criterion for the "self-duality" of $B_{+}$(see $(3.6))$.

1. The duality map

Let $F$ be the duality map of $B$. By definition 


$$
F(a)=\left\{f \in B^{*}:(f, a)=\|a\|^{2},\|f\|=\|a\|\right\}
$$

for every $a \in B$. It has the following properties:

(FI) $F(a)$ is a nonempty, closed and convex subset of $B^{*}$;

(F2) $F(-a)=-F(a)$;

(F3) $F(\lambda a)=\lambda F(a)$ for $\lambda \geq 0$;

(F4) for a bounded subset $A$ of $B, F(A)$ is a bounded subset of $B^{*}$;

(F5) $F$ is monotnoe, $(f-g, a-b) \geq 0$ when $f \in F(a)$ and $g \in F(b)$;

(F6) $F$ is demicontinuous: if $a_{n} \rightarrow a$ in $B$ and $f_{n} \in F\left(a_{n}\right)$, there exists $f \in F(a)$ such that $f_{n}(x)+f(x)$ for all $x \in B$;

(F7) $F$ is the subdifferential of the function $\mu(x)=\|x\|^{2} / 2$, that is, $F(a)=\partial \mu(a)$ for all $a \in B$;

(F8) if $B^{*}$ is strictly convex, $F$ is single-valued and strictly monotone: $(F(a)-F(b), a-b)=0$ implies $F(a)=F(b)$;

(F9) for every $a \in B$ and $b \in B$, there exist $a_{s}^{*} \in F(a)$ and $a_{i}^{*} \in F(a)$ such that

$$
\left(a_{s}^{*}, b\right)=\sup \{(f, b): f \in F(a)\}=\|a\|_{+}(a, b)
$$

and

$$
\left(a_{i}^{*}, b\right)=\inf \{(f, b): f \in F(a)\}=\|a\| \tau_{-}(a, b),
$$

where

$$
\tau_{+}(a, b)=\inf _{t>0} t^{-1}(\|a+t b\|-\|a\|)
$$

and

$$
\tau_{-}(a, b)=\sup _{t<0} t^{-1}(\|a+t b\|-\|a\|)
$$


For the proofs of these properties see, for instance [8], p. 119 and [9].

For the canonical half-norm $N$ of the positive cone $B_{+}$, we set

$$
H(a)=\left\{f \in B_{+}^{*}:(f, a)=N(a)^{2} \text { and }\|f\|=N(a)\right\} .
$$

Then it has the following properties:

(HI) $H(a)$ is a nonempty, closed and convex subset of $B_{+}^{*}$;

(H2) $H(a)=\{0\}$ if and only if $a \leq 0$;

(H3) $H(\lambda a)=\lambda H(a)$ for $\lambda \geq 0$;

(H4) $H$ is bounded;

(H5) $H$ is monotone;

(H6) $H$ is demicontinuous;

(H7) $H(a)=\partial v(a)$ for $v(x)=N(x)^{2} / 2$;

(H8) if $B_{+}^{*}$ is strictly convex, that is, if the relation $\|f+g\|=\|f\|+\|g\|$ for some $f, g \in B_{+}^{*}$ implies $f=\alpha g$ for some $\alpha$, then $H$ is single-valued and strictly monotone;

(H9) for every $a \in B$ and $b \in B$, there exist $a_{S}^{*} \in H(a)$ and $a_{i}^{*} \in H(a)$ such that

$$
\left(a_{s}^{*}, b\right)=\sup \{(f, b): f \in H(a)\}=N(a) \theta_{+}(a, b)
$$

and

$$
\left(a_{i}^{*}, b\right)=\inf \{(f, b): f \in H(a)\}=N(a) \theta_{-}(a, b),
$$

where

$$
\theta_{+}(a, b)=\inf _{t>0} t^{-1}(N(a+t b)-N(a))
$$

and

$$
\theta_{-}(a, b)=\sup _{t<0} t^{-1}(N(a+t b)-N(a))
$$

The property (H2) is an immediate consequence of the equivalent of 
$N(a)=0$ and $a \leq 0$. The other properties can be proved in the same manner as in the case of the duality map.

The map $H$ is used to study the order properties of the duality map $F$. The following statement is fundamental.

(1.1). For each $a \in B$, the following conditions are equivalent:

(1) $F(\alpha) \cap B_{+}^{*}=H(\alpha)$;

(2) $F(a) \cap B_{+}^{*} \neq \varnothing ;$

(3) $\|a\|=N(a)$.

Proof. (1) $\Rightarrow(2)$. This is obvious because $H(a) \neq \varnothing$.

(2) $\Rightarrow(3)$. For $f \in F(a) \cap B_{+}^{*}$,

$$
\|a\|^{2}=f(a) \leq\|f\| N(a)=\|a\| N(a) .
$$

Hence $\|a\|=N(a)$.

and $H(a)$.

$(3) \Rightarrow(1)$. This follows immediately from the definitions of $F(a)$

The duality map $F$ is said to be positive if $F(\alpha) \cap B_{+}^{*} \neq \emptyset$ for every $a \in B_{+}$.

(1.2). The following conditions are equivalent:

(1) $F$ is positive;

(2) $F(a) \cap B_{+}^{*}=H(a)$ for every $a \in B_{+}$;

(3) the norm of $B$ is monotone;

(4) the dual $B^{*}$ is $N_{+}$-decomposable;

(5) for $a \in B_{+}$and $f \in F(a)$, there exists $g \in F(a) \cap B_{+}^{*}$ such that $g \geq f$.

Proof. The equivalence of (1), (2) and (3) is an immediate consequence of (1.1) and the fact that the norm is monotone if and only if $\|a\|=N(a)$ for every $a \in B_{+}$. The equivalence of (3) and (4) has been proved in [11], Theorem 3.8. We shall prove that (4) implies (5). Let $a \in B_{+}$and $f \in F(a)$. By (4) there exist $g, h \in B_{+}^{*}$ such that $f=g-h^{\prime}$ and $N(f)=\|g\|$. Then, since $\|g\| \leq\|f\|$, 


$$
\begin{aligned}
0 \leq h(a) & =g(a)-f(a)=\|g\|\|a\|-\|a\|^{2} \\
& \leq\|f\|\|a\|-\|a\|^{2}=0 .
\end{aligned}
$$

Hence $g(a)=f(a)=\|a\|^{2}$ and $\|g\|=\|a\|$. Thus $g \in F(a) \cap B_{+}^{*}$. It is obvious that (5) implies (1).

The duality map $F$ is said to be inversely positive if $F(a) \cap B_{+}^{*} \neq \emptyset$ implies $a \in B_{+}$. Again, the following statement follows immediately from (1.1).

(1.3). The following conditions are equivalent;

(1) $F$ is inversely positive;

(2) if $N(a)=\|a\|$, then $a \in B_{+}$.

EXAMPLE 1. Suppose that $B$ is a Hilbert space $H$ with the positive cone $H_{+}$. Then the duality map is positive if and only if $H_{+} \subset H_{+}^{*}$, where $H_{+}^{*}=\left\{a \in H:(a, x) \geq 0\right.$ for every $\left.x \in H_{+}\right\}$, and the duality map is inversely positive if and only if $H_{+} \supset H_{+}^{*}$. When $H_{+}=H_{+}^{*}$, the positive cone has been called self-dual (see, for instance, [4], p. 104). The positive cone of the Banach lattice $L^{2}(\Omega)$ on a measure space $\Omega$, with the usual order, is obviously self-dual. In the Sobolev space $H_{0}^{\mathcal{I}}(\Omega)$, where $\Omega$ is a bounded domain with smooth boundary, equipped with the inner product

$$
(a, b)=\int_{\Omega} a^{\prime}(t) b^{\prime}(t) d t .
$$

the cone $K=\left\{a \in H_{0}^{\perp}(\Omega): a(t) \geq 0\right.$ almost everywhere $\}$ is closed and proper, and $H_{0}^{l}(\Omega)$ is a vector lattice. In this case $F=-\Delta$ in the distributional sense and the cone $K$ is not self-dual. However, $\left\{H_{0}^{l}(\Omega)\right]_{+}=\left\{a \in H_{0}^{l}(\Omega): a^{\prime}(t) \geq 0\right.$ almost everywhere $\}$ is a self-dual cone. Let $H_{n}$ be the linear space of all $n$-square hermitian matrices equipped with the inner product $(a, b)=$ trace of $b a$. Then $\left(H_{n}\right)_{+}=\left\{a \in H_{n}: a\right.$ is positive semi-definite $\}$ is a self-dual cone (see [1]. p. 70). This is also true for general operator algebras with traces. 
For more general cases, see [4].

Following this example, we shall call the positive cone $B_{+}$of an ordered Banach space $B$ self-dual if the duality map is positive and inversely positive. Then the following statement is obvious.

(1.4). The following conditions are equivalent:

(1) $B_{+}$is self-dual;

(2) $N(a)=\|a\|$ if and only if $a \in B_{+}$.

EXAMPLE 2. The norm of $B$ is said to be of type $(N, p)$,

$1 \leq p \leq \infty$, if

$$
\|a\|^{p}=N(a)^{p}+N(-a)^{p} \text { if } p \text { is finite }
$$

and

$$
\|a\|=\max (N(a), N(-a)) \text { if } p=\infty .
$$

(See [13], §6.) It is obvious that, if the norm is of type $(N, p)$ for finite $p$, the positive cone is self-dual. When $p=\infty$, the duality map is positive but not inversely positive. When $B$ is the hermitian part of a $C^{*}$-algebra with the usual order, it then follows that $B_{+}$is not selfdual but the dual cone $B_{+}^{*}$ is self-dual.

EXAMPLE 3 . Let $B$ be the Banach space $c^{1}[0,1]$ equipped with the norm $\|a\|=\sup _{0 \leq t \leq 1}|a(t)|+\sup _{0 \leq t \leq 1}\left|a^{\prime}(t)\right|$. Define the positive cone by

$$
B_{+}=\left\{a \in C^{l}[0,1]: a(t) \geq 0 \text { for all } t\right\} \text {. }
$$

Then $N(a)=\sup _{0 \leq t \leq 1} a_{+}(t)$, and $N(a)=\|a\|$ if and only if $a$ is a positive number. Thus the duality map is neither positive nor inversely positive.

As we have shown in (1.2), the positivity of $F$ has a simple characterization that the norm is monotone. However, a simple characterization of the inverse positivity of $F$ has to wait until we have the notion of orthogonally decomposable spaces (see (3.5)). The following two statements are concerned with this problem in general cases.

(1.5). Suppose that $B$ is reflexive and strictly convex. Then the following conditions are equivalent: 
(1) $F$ is inversely positive;

(2) the dual norm is monotone.

Proof. (1) $\Rightarrow(2)$. Let $f \in B_{+}^{*}$ and $F^{*}$ be the duality map of $B^{*}$. Suppose that $\xi \in F^{*}(f)$. Since $B$ is reflexive, $\xi=\hat{a}$ for some $a \in B$, where $(\hat{a}, g)=(g, a)$ for all $g \in B^{*}$. Then $f \in F(a)$ and, by (I), $a \in B_{+}$. Furthermore,

$$
\|f\|^{2}=\|a\|^{2}=(f, a) \leq N(f)\|a\| \text {. }
$$

Hence $\|f\|=N(f)$. Therefore the dual norm is monotone.

(2) $\Rightarrow(1)$. Suppose that $f \in F(a) \cap B_{+}^{*}$. Then, by (2), it follows from (1.2) that there exists $\xi \in F^{*}(f) \cap B_{+}^{* *}$. However, since $B$ is reflexive and strictly convex, $F^{*}$ is single-valued. Hence, since $\hat{a} \in F^{*}(f)$, we have $\xi=\hat{a}$ and this implies $a \in B_{+}$.

Therefore, when $B$ is reflexive and strictly convex, the positive cone $B_{+}$is self-dual if and only if the norms of $B$ and $B^{*}$ are monotone.

When $B$ is a Banach lattice, $\|a\|=N(a)$ is equivalent to $\left\|a_{+}\right\|=\|(|a|)\|$. Therefore, in this case, $F$ is inversely positive if and only if $\left\|a_{+}\right\|=\|(|a|)\|$ implies $a_{+}=|a|$. Generally, the norm of an ordered Banach space $B$ is said to be strictly monotone if $\|a\|<\|b\|$ when $0 \leq a \leq b$ and $a \neq b$.

(1.6). Suppose that the norm of $B^{*}$ is smooth. Then, if the norm of $B$ is monotone, it is strictly monotone.

Proof. Suppose that $0 \leq a \leq b$ and $\|a\|=\|b\|$. Since the norm is monotone, $F$ is positive by (1.2). Hence there exist $f \in F(a) \cap B_{+}^{*}$ and $g \in F(b) \cap B_{+}^{*}$. Then $(g, b)=\|b\|^{2}$ and

$$
\|a\|^{2}=(f, a) \leq(f, b) \leq\|f\|\|b\|=\|b\|^{2} .
$$

Therefore $(f, a)=(f, b)=(g, b)$. On the other hand, since $F^{*}$ is single-valued, we have $F^{*}(f)=\hat{a}$ and $F^{*}(g)=\hat{b}$. Therefore $\left(F^{*}(g), f\right)=\|f\|^{2}$ and $\left\|F^{*}(g)\right\|=\|f\|$, and hence, $F^{*}(g)=F^{*}(f)$. Thus we have $a=b$.

It follows from (1.6) that the positive cone of a Banach lattice with 
smooth dual is self-dual.

The following relation between $F$ and $H$ is fundamental in the subsequent arguments.

(1.7). If $b \geq a$ and $N(a)=\|b\|$, then

$$
H(a)=F(b) \cap B_{+}^{*} \cap(b-a)^{0} \text {, }
$$

where $(b-a)^{0}=\left\{f \in B^{*}:(f, b-a)=0\right\}$.

Proof. When $f \in H(\alpha)$, we have $f \in B_{+}^{*}$ and

$$
\|b\|^{2}=N(a)^{2}=(f, a) \leq(f, b) \leq\|f\|\|b\|=N(a)\|b\|=\|b\|^{2} .
$$

Hence $f \in F(b)$ and $(f, b-a)=0$. Therefore $H(a)$ is contained in the right-hand side. The other inclusion is obvious.

There are cases when $F(b)$ in (1.7) is automatically contained in $B_{+}^{*}$. When $B$ is $N$-decomposable, every element $a$ is expressed as $a=a_{+}-a_{-}$, where $a_{ \pm} \in B_{+}, N(a)=\left\|a_{+}\right\|$and $N(-a)=\left\|a_{-}\right\|$. Therefore $a_{+}$satisfies the conditions placed on $b$ in (1.7).

(1.8). Suppose that $B$ is $N$-decomposable and the dual positive cone $B_{+}^{*}$ is self-duat. Then, for every $a \in B$, we have $F\left(a_{+}\right) \subset B_{+}^{*}$ and, therefore, $H(a)=F\left(a_{+}\right) \cap\left(a_{-}\right)^{0}$.

Proof. Let $f \in F\left(a_{+}\right)$. Since $B^{*}$ is also $N$-decomposable by [13], Theorem 5.2, we can take an $N$-decomposition $f=f_{+}-f_{-}$of $f$. Then

$$
\begin{aligned}
0 & \leq\left(f_{-}, a_{+}\right)=\left(f_{+}, a_{+}\right)-\left(f, a_{+}\right)=\left(f_{+}, a_{+}\right)-\left\|a_{+}\right\|^{2} \\
& \leq\left\|f_{+}\right\|\left\|a_{+}\right\|-\left\|a_{+}\right\|^{2}=N(f)\left\|a_{+}\right\|-\left\|a_{+}\right\|^{2} \\
& \leq\|f\|\left\|a_{+}\right\|-\left\|a_{+}\right\|^{2}=0,
\end{aligned}
$$

because $\|f\|=\left\|a_{+}\right\|$. Hence we have $N(f)=\|f\|$. Since the dual cone is self-dual, we have $f \in B_{+}^{*}$.

When $B$ is the hermitian part of a $C^{*}$-algebra, then, as was stated in Example 2, $B$ is $N$-decomposable and $B_{+}^{*}$ is self-dual. Therefore the assumptions in (1.9) are satisfied.

When an ordered Banach space $B$ is $N$-decomposable and $a \in B_{+}$, then $a=a-0$ is the only $N$-decomposition. Hence the following statement follows immediately from (1.8). For the case of the hermitian part of a 
$C^{*}$-algebra, see [3], Example 1.2.5.

(1.9). Suppose that $B$ is $N$-decomposable and $B_{+}^{*}$ is self-dual. Then $F(a) \subset B_{+}^{*}$ for every $a \in B_{+}$.

\section{Projections}

For $a \in B$, we set

$$
\pi(a)=\left\{b \in B_{+}:\|a-b\| \leq\|a-x\| \text { for all } x \in B_{+}\right\},
$$

which will be called the projection of $a$ on the positive cone $B_{+}$.

It is obvious that $a \in \pi(a)$ if $a \in B_{+}, \pi(\lambda a)=\lambda \pi(a)$ for $\lambda \geq 0$, and $\pi(\pi(a))=\pi(a)$ for every $a \in B$. Furthermore, it is easy to see that $0 \in \pi(a)$ whenever $a \leq 0$ if and only if the norm of $B$ is monotone.

(2.1). The following conditions are equivalent:

(1) $b \in \pi(a)$;

(2) $\|b-a\|=N(-a)$ and $b \in B_{+}$;

(3) $b \in B_{+}$and $(f, b)=0$ for some $f \in F(b-a) \cap B_{+}^{*}$.

Proof. The equivalence of (1) and (2) is obvious from the definitions of $\pi$ and $N$. The fact that (2) implies (3) follows from (1.7), which implies $H(-a)=F(b-a) \cap B_{+}^{*} \cap(b)^{0}$. To prove that (3) implies (2), let $f \in F(b-a) \cap B_{+}^{*}$ and $(f, b)=0$. Then

$$
\|b-a\|^{2}=(f, b-a)=(f,-a) \leq\|f\| N(-a) .
$$

Thus $\|b-a\|=N(-a)$.

The set $\pi(a)$ may be empty. $B_{+}$is said to be projectable if $\pi(a)$ is not empty for every $a \in B$.

(2.2). The following conditions are equivalent:

(1) $B_{+}$is projectable;

(2) for every $a \in B$ there exists $b \in B$ such that $b \geq a$ and $\|b\|=N(a)$;

(3) $B_{1}-B_{+}$is closed.

Proof. (1) $\Rightarrow(2)$. For $a \in B$, take $c \in \pi(-a)$. Then, by (2.1), 
$b=a+c$ is the required element.

(2) $\Rightarrow$ (3). Let $a \in B(N)=\{x \in B: N(x) \leq 1\}$. Then, by (2), there exists $b \in B$ such that $b \geq a$ and $b \in B_{1}$. Hence $a \in B_{1}-B_{+}$. However $B(N)=\overline{B_{1}-B_{+}}$. Hence $B_{1}-B_{+}$is closed.

$(3) \Rightarrow(1)$. When $N(-a)=0$, then $a \in B_{+}$and, hence, $a \in \pi(a)$. When $N(-a) \neq 0, N(-a)^{-1}(-a) \in B(N)=B_{1}-B_{+}$; that is, $-a=N(-a) b-N(-a) c$ for some $b \in B_{1}$ and $c \in B_{+}$.

Since $N(-a) \leq N(-a) N(b)$ we have $\|b\|=1$. Then, since $N(-a) b=N(-a) c-a$, we have $N(-a)=\|N(-a) c-a\|$. Therefore, by (2.1), we have $N(-a) c \in \pi(a)$.

It is obvious that $B_{1}-B_{+}$is closed if $B_{1}$ is weakly compact. Hence the dual $B_{+}^{*}$ is always projectable.

The following statement, which is an immediate consequence of (2.2), shows that $N_{+}$-decomposable spaces are projectable. Therefore all Banach lattices and the hermitian parts of $C^{*}$-algebras are proiectable.

(2.3). The following conditions are equivalent:

(1) $B$ is $N_{+}$-decomposable;

(2) for every $a \in B$ there exists $b \in B_{+}$such that $b \geq a$ and $b \in \pi(a)$.

There are spaces which are projectable but not $N_{+}$-decomposable. For example, when the norm of $B$ is not monotone, the dual $B^{*}$ is not $N_{+}-$ decomposable although $B_{+}^{*}$ is always projectable.

(2.4). If $B$ is projectable and $F$ is inversely positive, then $B$ is $N_{+}$-decomposable.

Proof. For $a \in B$, take $b \in \pi(a)$. Then, since, by (2.1),

$$
N(b-a) \leq\|b-a\|=N(-a) \leq N(b-a),
$$

we have $N(b-a)=\|b-a\|$. Since $F$ is inversely positive, we then have $b \geq a$. Hence it follows from (2.3) that $B$ is $N_{+}$-decomposable.

It is easy to see that $\pi$ is single-valued if $B$ is strictly convex. The following statement is an immediate consequence of (2.3). 
(2.5). If $B$ is $N_{+}$-decomposable and $\pi$ is single-valued, then the $N_{+}$-decompositions are unique.

Suppose that $B$ is a Hilbert space $H$ considered in Example 2. Then it is projectable since the unit ball is weakly compact. Furthermore, $\pi$ is single-valued because $H$ is strictly convex. Hence, by (2.1) (3), for every $a \in H$ there exist $b \in H_{+}$and $c \in H_{+}^{*}$ such that $a=b-c$ and $(b, c)=0$. Therefore, if $H_{+}^{*} \subset H_{+}$, that is, if the duality map is inversely positive, $a=b-c, b \in H_{+}$and $c \in H_{+}$with $(b, c)=0$. This fact is generalized in the following form.

(2.6). Suppose that $B$ is projectable and $F$ is inversely positive. Then, for any $a \in B$, there exist $b \in B_{+}, c \in B_{+}$and $c^{*} \in F(c) \cap B_{+}^{*}$ such that $a=b-c$ and $\left(b, c^{*}\right)=0$.

Proof. For $a \in B$, let $b \in \pi(a)$. Then, by (2.1), $b \geq 0$ and $\left(b, c^{*}\right)=0$ for some $c^{*} \in F(c) \cap B_{+}^{*}$, where $c=b-a$. Since $F$ is inversely positive, $c \in B_{+}$.

We conclude this section with a simple remark which follows from (1.5) and $(2.3)$.

(2.7). When $B$ is a Hizbert space $H$, the following conditions are equivalent:
(1) $\mathrm{H}$ is $\mathrm{N}_{+}$-decomposable;
(2) $F$ is inversely positive;
(3) $\pi(a) \geq a$ for every $a \in H$.

\section{Orthogonal decompositions}

When $B$ is $N$-decomposable, every element $a$ can be expressed as $a=b-c$ for some $b, c \in B_{+}$such that $N(a)=\|b\|$ and $N(-a)=\|c\|$. These two positive elements $b$ and $c$ satisfy, therefore, the following two equations :

$$
N(b-c)=\|b\| \text { and } N(c-b)=\|c\| \text {. }
$$

Generally, two positive elements $b$ and $c$ will be called $N$-orthogonal if they satisfy these two equalities.

Therefore $a=b-c$ is an $N$-decomposition if and only if $b$ and $c$ are $N$-orthogonal. When $B$ is the hermitian part of a $C^{*}$-algebra and 
$b c+c b=0$, then $b$ and $c$ are $N$-orthogonal because the equality implies $(b-c)_{+}=b$ and $(c-b)_{+}=c$. However, there are other $\mathrm{N}-$ orthogonal elements which do not satisfy the equality as we shall see in Example 4. The case of Banach lattices is much simpler.

(3.1). When $B$ is a Banach lattice and $b \wedge c=0$, then $b$ and $c$ are N-orthogonal. The converse holds if the dual $B^{*}$ is smooth.

Proof. If $b \wedge c=0$ we have $(b-c)_{+}=b$ and, therefore, $N(b-c)=\|b\|$. Similarly, $N(c-b)=\|c\|$. Conversely, when $b$ and $c$ are $N$-orthogonal, since we always have $0 \leq(b-c)_{+} \leq b$, it follows from $\left\|(b-c)_{+}\right\|=\|b\|$ and $(1.6)$ that $(b-c)_{+}=b$. Therefore $b \wedge c=0$.

Two positive elements $b$ and $c$ are said to be $F$-orthogonal if there exist $b^{*} \in F(b) \cap B_{+}^{*}$ and $c^{*} \in F(c) \cap B_{+}^{*}$ such that $\left(b^{*}, c\right)=\left(b, c^{*}\right)=0$.

(3.2). For $b \in B_{+}$and $c \in B_{+}$, the following conditions are equivalent:

(1) $b$ and $c$ are F-orthogonal;

(2) $b$ and $c$ are N-orthogonal;

(3) $b \in \pi(b-c)$ and $c \in \pi(c-b)$.

Proof. The equivalence of (2) and (3) is an immediate consequence of (2.1).

$(1) \Rightarrow(2)$. For the functional $b^{*}$ in the definition of $F-$ orthogonality,

$$
\begin{aligned}
N(b-c) & =\sup \{(f, b-c): f \geq 0,\|f\| \leq 1\} \\
& \geq\|b\|^{-1}\left(b^{*}, b-c\right)=\|b\| .
\end{aligned}
$$

Hence we have $N(b-c)=\|b\|$. Similarly, $N(c-b)=\|c\|$.

(3) $\Rightarrow(1)$. This follows immediately from (2.1).

Two positive elements $b$ and $c$ will simply be called orthogonal if they satisfy one of the three equivalent conditions in (3.2), and we shall denote this fact by $b \perp c$. Since $F(\lambda a)=\lambda F(a)$ for $\lambda \geq 0, b \perp c$ implies $b \perp \lambda c$ for $\lambda \geq 0$.

When $B$ is a Hilbert space and the norm is monotone, $b \perp c$ is equivalent to $(b, c)=0$. When $B$ is a Banach lattice with smooth dual, 
it follows from (3.1) that $b \perp c$ is equivalent to $b \wedge c=0$. Therefore, in these two cases, we have that $b \perp c$ and $0 \leq d \leq c$ imply $b \perp d$. However, this is not always true.

EXAMPLE 4. Let $B=C[-1,1]$ be the Banach lattice of continuous functions on $[-1,1]$ with the pointwise order, and consider the function $a(t)=t$. Then, in addition to the lattice decomposition $a=a_{+}-a_{-}$, there are infinitely many orthogonal decompositions in our sense. For example, $a=b-c$ is an orthogonal decomposition when $b$ is the line connecting the points $(-1,0)$ and $(1,1)$, and $c$ is the line connecting $(-1,1)$ and $(1,0)$. It is clear that there exists $d \in B_{+}$ such that $d \leq c$ but $b \perp d$ is not satisfied. Note also that $\pi(a)=\left\{x \in C[-1,1]: a_{+} \leq x \leq e\right\}$, where $e(t)=t+1$. The fact that $a_{+}$is the smallest element of $\pi(a)$ is not accidental, as we shall see in $(3.5)$.

The following statement is a partial answer to this question.

(3.3). Suppose that the norm of $B$ is monotone. If $b \perp c$, then $b \perp d$ when there exists $\alpha>0$ such that $\alpha c \geq d \geq 0$ and $N(\alpha c-b)=N(d-b)$.

Proof. Since $b \perp a c$ and the norm is monotone, $N(\alpha c-b)=\|\alpha c\| \cdot \geq\|d\| \geq N(d-b)$.

Hence $N(d-b)=\|d\|$. On the other hand, since

$$
\|b\|=N(b-\alpha c) \leq N(b-d) \leq\|b\|,
$$

we have $N(b-d)=\|b\|$. Therefore $b \perp d$.

An ordered Banach space $B$ is called orthogonally decomosable if every element $a$ is expressed as $a=b-c$ such that $b \perp c$. Then the following statement is obvious.

(3.4). The following conditions are equivalent:

(1) $B$ is N-decomposable;

(2) $B$ is orthogonally decomposable;

(3) $a \in \pi_{0}(a)-\pi_{0}(-a)$ for every $a \in B$, where

$$
\pi_{0}(a)=\{b \in \pi(a): b \geq a \text { and }\|b\|=N(a)\} .
$$


Therefore all Banach lattices and all hermitian parts of $C^{*}$-algebras are orthogonally decomposable. Throughout the remainder of this paper, we shall always assume that $B$ is orthogonally decomposable. Hence, when we set $\left(a_{+}\right)=\pi_{0}(a)$ and $\left(a_{-}\right)=\pi_{0}(-a)$, we have

$$
a \in\left(a_{+}\right)-\left(a_{-}\right) \text {for every } a \in B \text {. }
$$

The following statement can be proved easily. The set $\left(a_{+}\right)$will sometimes be denoted simply by $a_{+}$.

(3.5). (1) $b \in\left(a_{+}\right)$if and only if $b-a \in\left(a_{-}\right)$.

(2) If $a \in B_{+}$, then $\left(a_{-}\right)=\{0\}$.

(3) $\left(a_{+}\right)=\{a\}$ for alz $a \in B_{+}$.

(4) For $b \in\left(a_{+}\right),\left(b_{+}\right)=\left(a_{+}\right) \cap\{x \in B: x \geq b\}$.

(5) If $b \perp c, b \in(b-c)_{+}$and $c \in(b-c)_{-}$.

(6) If $B$ is a Banach lattice and $a=a_{+}-a_{-}$is the lattice decomposition, then $a_{+} \leq b$ for all $b \in\left(a_{+}\right)$.

Since $\|b\|=N(a)$ for every $b \in\left(a_{+}\right)$, we can define the norm $\left\|a_{+}\right\|$ of the set $\left(a_{+}\right)$by this common value; that is, $\left\|a_{+}\right\|=N(a)$. Then we have a characterization of the self-duality of $B_{+}$when $B$ is orthogonally decomposable. Note that the norm of an orthogonally decomposable space is monotone, and, hence, the duality map is always positive.

(3.6). The folzowing conditions are equivalent:

(1) $B_{+}$is self-dual;

(2) $\|a\|=\left\|a_{+}\right\|$implies $a \in B_{+}$:

(3) if $b \perp c$ and $\|b\|=\|b-c\|$, then $c=0$.

Proof. The equivalence of (1) and (2) follows from $\left\|a_{+}\right\|=N(a)$. When we set $a=b-c$, conditions (2) and (3) are identical.

REMARK. The self-duality of $B_{+}$is determined by $B_{+}$and the norm of $B$. To produce a self-dual cone from a positive cone which is not, one usually retains the norm and reforms $B_{+}$, as in the case of operator algebras. We note here that it is always possible to make the original $B_{+}$ self-dual by changing the norm. More precisely, when $B$ is orthogonally 
decomposable, there is an equivalent norm on $B$ so that $B_{+}$is self-dual. To prove this, one only has to considex the norm $\|\mid a\|=\left(N(a)^{p}+N(-a)^{p}\right)^{1 / p}$ for an arbitrary finite $p$. Since the original norm is monotone, the two norms are equivalent. The canonical half-norm of $B_{+}$measured by the new norm coincides with the original $N$ because of the orthogonal decomposability. The self-duality of $B_{+}$is now obvious.

We now leave the self-duality and consider another problem. Suppose that $B$ is a Hilbert space $H$ and let $K_{a}=\{x \in H: x \leq a\}$. Then $K_{a}$ is a convex closed subset of $H$ and its indicator function

$$
I_{a}(x)=0 \text { when } x \in K_{a},=+\infty \text { otherwise, }
$$

is a proper lower-semicontinuous convex function. The subdifferential $\partial I_{a}(c)$ is of the following form:

$$
\partial I_{a}(c)=\left\{x \in H_{+}: x\lfloor a-c\} .\right.
$$

It is known [8] that, when $H$ is the space $L^{2}(\Omega)$, lattice-ordered in the usual manner, the resolvent of $\partial I_{a}$ is single-valued and

$$
\left(1+\lambda \partial I_{a}\right)^{-1}(b)=a \wedge b
$$

for all $\lambda>0$. A similar equality holds in $L^{p}(\Omega), 1 \leq p<\infty$, with suitable modifications.

Returning to the case of general orthogonally decomposable spaces, we set

$$
a \wedge b=a-(a-b)_{+}\left(=b-(b-a)_{+}\right)
$$

and

$$
a \vee b=a+(a-b)_{-}\left(=b+(b-a)_{-}\right) .
$$

Then it is easy to see that

$$
a+b \in a \vee b+a \wedge b
$$

Now, for $a \in B$, we define a relation $A_{a} \subset B \times B$ by

$$
A_{a}=\{(c, x): c \leq a, x \geq 0 \text { and } x \perp a-c\} \text {. }
$$


(3.7). $\left(1+\lambda A_{a}\right)^{-1}(b)=a \wedge b$ for every $a, b \in B$ and $\lambda>0$.

Proof. By the definition,

$$
1+\lambda A_{a}=\{(c, b): c \leq a, c \leq b, b-c \perp a-c\} \text {. }
$$

Hence, by $(3.4)$,

$$
\begin{aligned}
\left(1+\lambda A_{a}\right)^{-1}(b) & =\{c: c \leq a, c \leq b, b-c \perp a-c\} \\
& =\left\{c: a-c \in(a-b)_{+}\right\} \\
& =a \wedge b .
\end{aligned}
$$

Thus the resolvent $\left(1+\lambda A_{a}\right)^{-1}$ is not single-valued in general. The following statement can be proved easily.

(3.8). The following conditions are equivalent:

(1) $\left(1+\lambda A_{a}\right)^{-1}$ is single-valued:

(2) $\pi_{0}$ is single-valued;

(3) $B$ is zoniquely orthogonally decomposable.

We note that, when $B$ is a Hilbert space, it is uniquely orthogonally decomposable if and only if $B_{+}$is self-dual.

In the sequel we shall assume that $B$ is coniquely orthogonally decomposable. Then, since

$$
a \wedge b-a \wedge c=(a-c)_{+}-(a-b)_{+},
$$

$\left(1+\lambda A_{a}\right)^{-1}$ is a norm-contraction if and only if the single-valued map $\pi_{0}$ is. When $B$ is a Banach lattice or a Hilbert space with self-dual positive cone, $\pi$ is, and hence $\pi_{0}$ is, a norm-contraction.

In fact, in the latter case, we have

$$
(\pi(a)-\pi(b), a-b) \geq 0 \text { for all } a, b \in B \text {. }
$$

However, in the general case, in order that $\pi_{0}$ be an $N$-contraction, that is,

$$
N\left(\pi_{0}(a)-\pi_{0}(b)\right) \leq N(a-b) \text { for all } a, b \in B \text {, }
$$


$B$ must be a vector lattice as the following statement shows.

(3.9). The following conditions are equivalent:

(1) $\pi_{0}$ is an N-contraction;

(2) $a \leq b$ implies $\pi_{0}(a) \leq \pi_{0}(b)$;

(3) $B$ is a vector lattice with respect to the original order.

Proof. (1) $\Rightarrow(2)$. This follows from the equivalence of $N(-a)=0$ and $a \in B_{+}$.

(2) $\Rightarrow(3)$. Suppose that $a \leq b$ and $b \in B_{+}$. Then $a_{+}=\pi_{0}(a) \leq \pi_{0}(b)=b$. Therefore $a_{+}=\sup \{a, 0\}$. This shows that $B$ is a vector lattice.

$(3) \Rightarrow(1)$. When $B$ is a vector lattice,

$$
\begin{aligned}
\left(a_{+}-b_{+}\right)_{+}=\left(a \vee 0-b_{+}\right) & =\left(a-b_{+}\right) \vee\left(-b_{+}\right) \vee 0 \\
& \leq(a-b) \vee 0=(a-b)_{+} .
\end{aligned}
$$

Therefore

$$
\begin{aligned}
N\left\{\pi_{0}(a)-\pi_{0}(b)\right\} & =N\left(a_{+}-b_{+}\right)=\left\|\left(a_{+}-b_{+}\right)_{+}\right\| \\
& \leq\left\|(a-b)_{+}\right\|=N(a-b) .
\end{aligned}
$$

because the norm is monotone.

REMARK 1. There is a notion of orthogonality in a general Banach space $B$ due to R.C. James. According to [6], an element $a$ of $B$ is said to be James-orthogonal to an element $b$ if $\|a\| \leq\|a+\lambda b\|$ for all $\lambda \in R$. It is easy to see that this is equivalent to $(f, b)=0$ for some $f \in F(a)$. Hence it is clear that, if $a \in B_{+}$and $b \in B_{+}$are orthogonal in our sense, then $a$ is James-orthogonal to $b$ and $b$ is Jamesorthogonal to $a$. The converse holds if the assumptions in (1.9) are satisfied.

REMARK 2. There are several related topics which have not been discussed in this note. These include the absolute monotonicity of the norms, the absolute values of elements and the Robinson property in orthogonally decomposable spaces. It serves to clarify these properties if we consider an example, which appeared in [7]. Let $K$ be the twodimensional space $R^{2}$ with the usual coordinatewise order and the norm 


$$
\|a\|=|x+y| \text { if } x y \geq 0,=\left(x^{2}+y^{2}\right)^{\frac{1}{2}} \text { otherwise }
$$

for $a=(x, y)$. Then the dual $K^{*}$ is $R^{2}$ with the same order and the norm

$$
\|a\|=|x| \vee|y| \text { if } x y \geq 0,=\left(x^{2}+y^{2}\right)^{\frac{1}{2}} \text { otherwise. }
$$

It is easy to see that $K$ and $K^{*}$ are uniquelv orthogonally decomposable, both are of type $(N, 2)$ and both have self-dual positive cones. However, although the norm of $K$ is absolutely monotone, the norm of $K^{*}$ is not. Nevertheless, it follows from [13], (7.2), that $K$ and $K^{*}$ have the Robinson property, that is, for every positive continuous linear operator $A$, we have $\|A\|=\sup \left\{\|A a\|:\|a\| \leq 1\right.$ and $\left.a \in B_{+}\right\}$. Therefore, these spaces are examples of spaces with the Robinson property whose norms are not Riesz norms (see [10]). As to the absolute values, we observe that $\|a\|<\left\|a_{+}+a_{-}\right\|$in $K$ and $\|a\|>\left\|a_{+}+a_{-}\right\|$in $K^{*}$ for the unique orthogonal decomposition $a=a_{+}-a_{-}$of an element $a$ which is neither positive nor negative.

\section{Automorphisms}

Throughout this section we shall always assume that $B$ is an orthogonally decomposable space. A linear operator $S: B \rightarrow B$ is called a transition map if $\|S b\|=\|b\|$ for all $b \in B_{+}$. An automorphism is a bijective transition map $S: B \rightarrow B$ which preserves the orthogonality, that is, $a=b-c$ is an orthogonal d-composition if and only if $S a=S b-S c$ is an orthogonal decomposition. It is obvious that automorphisms are continuous.

The $N$-preserving linear operators have been considered in [14]. A bijection $S: B \rightarrow B$ is $N$-preserving if $N(S a)=N(a)$ for every $a \in B$.

(4.1). The following conditions are equivalent for a bijection $S: B \rightarrow B:$

(1) $S$ is an automorphism;

(2) $S$ is $N$-preserving;

(3) $S^{\star} H(S a)=H(a)$ for every $a \in B$.

Proof. (1) $\Rightarrow(2)$. For $a \in B$, let $a=b-c$ be an orthogonal 
decomposition. Then, by the assumption, $S a=S b-S c$ is also an orthogonal decomposition. Since $S$ is a transition map and $b \in S_{+}$, $N(S a)=\|S b\|=\|b\|=N(a)$.

$$
\begin{array}{r}
(2) \Rightarrow(3) \text {. Let } f \in H(S a) \text {. Then, since } f \in B_{+}^{*}, \\
\left(S^{*} f, x\right)=(f, S x) \leq\|f\| N(S x)=\|f\| N(x)
\end{array}
$$

for every $x \in B$. Therefore

$$
\left\|S^{*} f\right\| \leq\|f\|=N(S a)=N(a) .
$$

On the other hand,

$$
\left(S^{*} f, a\right)=(f, S a)=N(S a)^{2}=N(a)^{2},
$$

and this also implies $\left\|S^{*} f\right\| \geq N(a)$. Thus $\left\|S^{*} f\right\|=N(a)$. The positivity of $S$, and of $S^{-1}$, follows immediately from (2). Hence $S^{*} f \in H(a)$, and we have proved that $S^{*} H(S a) \subset H(a)$. We obtain the inverse inclusion by replacing $S$ with $S^{-1}$.

$(3) \Rightarrow(1)$. At first we note that $S$ is positive, because $H(S a)=\{0\}$ (that is, $S a \leq 0$ ) if $a \leq 0$. Now, for $b \in B_{+}$, let $f \in H(S b)$. Then, since $S^{*} f \in H(b)$,

$$
\|b\|^{2}=N(b)^{2}=\left(S^{*} f, b\right)=(f, S b)=N(S b)^{2}=\|S b\|^{2} .
$$

Therefore $S$ is a transition map. Next, suppose $b \perp c$, and choose $b^{*} \in H(a)$ and $c^{*} \in H(-a)$ such that $\left(b^{*}, c\right)=\left(b, c^{*}\right)=0$, where $a=b-c$. Then $b^{*}=S^{*} f$ and $c^{*}=S^{*} g$ for some $f \in H(S a)$ and $g \in H(-S a)$. It then follows that

$$
\begin{aligned}
N(S a)^{2} & =(f, S a)=\left(S^{*} f, a\right)=\left(b^{*}, a\right)=\left(b^{*}, b\right)=\|b\|^{2} \\
& =\|S b\|^{2},
\end{aligned}
$$

because $S$ is a transition map. Similarly, $N(-S a)=\|S c\|$. Hence $S b \perp S c$. Considering $S^{-1}$ instead of $S$, we have that $S b \perp S c$ implies $b \perp c$.

(4.2). If $S$ is an automorphism of $B$, then $S^{*}$ is an automorphism of $B^{*}$.

Proof. Obviously $S^{*}$ is a bijection. Furthermore, since $S$ is a positive transition map, for every $f \in B^{*}$, 


$$
\begin{aligned}
N\left(S^{*} f\right) & =\sup \left\{\left(S^{*} f, x\right): x \in B_{+},\|x\| \leq 1\right\} \\
& =\sup \left\{(f, y): y \in B_{+},\|y\| \leq 1\right\}=N(f) .
\end{aligned}
$$

Hence $S^{*}$ is an automorphism.

We now characterize automorphisms of some important class of orthogonally decomposable spaces. As we have stated in the remark after (3.9), the norm of an ordered Banach space $B$ is said to have the Robinson property if

$$
\|S\|=\|S\|_{+}=\sup \left\{\|S a\|: a \in B_{+},\|a\| \leq 1\right\}
$$

for every positive continuous linear operator $S: B \rightarrow B$. It is known that the norms of all Banach lattices and the hermitian parts of $C^{*}$ algebras have the Robinson property.

(4.3). Suppose that the norm of $B$ has the Robinson property and $S: B \rightarrow B$ is a bijection. Then the following conditions are equivalent:

(1) $S$ is an automorphism;

(2) $S$ and $S^{-1}$ are positive and isometric, that is, $\|S a\|=\|a\|$ for every $a \in B$;

(3) $S$ and $S^{-1}$ are positive and $S^{*} F(S a)=F(a)$ for every $a \in B$.

Proof. Generally a bijection $S$ of a Banach space $B$ is an isometry if and only if $S^{*} F(S a)=F(a)$ for all $a \in B$. Hence (2) and (3) are equivalent. We shall prove the equivalence of (1) and (2).

(1) $\Rightarrow(2)$. By (4.1), $\|S\|_{+}=1$. Hence it follows from the Robinson property that $\|S a\| \leq\|a\|$ for every $a \in B$. Similarly, we have $\left\|S^{-1} a\right\| \leq\|a\|$, that is, $\|a\| \leq\|S a\|$ for every $a \in B$. Therefore $S$ is an isometry.

$(2) \Rightarrow(1)$. It follows from the assumption that the dual $S^{*}$ is also an isometry of $B^{*}$. Therefore the condition that $f \in B_{+}^{*}$ and $\|f\| \leq 1$ is equivalent so that $S^{*} f \in B_{+}^{*}$ and $\left\|S^{*} f\right\| \leq 1$. Hence, for every $a \in B$,

$$
\begin{aligned}
N(S a) & =\sup \left\{(f, S a): f \in B_{+}^{*},\|f\| \leq 1\right\} \\
& =\sup \left\{\left(S^{*} f, a\right): S^{*} f \in B_{+}^{*},\left\|S^{*} f\right\| \leq 1\right\} \\
& =N(a) .
\end{aligned}
$$


Therefore $S$ is an automorphism.

Therefore, when $B$ is a Banach lattice, $S$ is an automorphism if and only if it is an isometric lattice-isomorphism. When $B$ is the hermitian part of a unital $C^{*}$-algebra acting nondegenerately on a Hilbert space, it follows from [4], Theorem 3.2.3, that $S$ is an automorphism such that $S(1)=1$ if and only if it is the restriction on $B$ of a Jordan automorphism of the algebra. In general $C^{*}$-algebras, a $C_{0}$-group of Jordan automorphisms is in fact a group of *-automorphisms. (See [4], Corollary 3.2 .12$.

(4.4). Let, as before, $B$ be an orthogonally decomposable space. The following two conditions are equivalent:

(1) $G$ is a generator of a $C_{0}$-group $\left\{S_{t}: t \in R\right\}$ of automorphisms;

(2) (a) $G$ is densely defined,

(b) $R(1+\alpha G)=R(1+\beta G)=B$ for some $\alpha>0$ and $\beta>0$,

(c) $(f, G a)=0$ if $a \in D(G)$ and $f \in H(a)$.

Proof. It has been proved in [3], Corollary 2.2.5, that, under the assumption that the norms of $B$ and $B^{*}$ are monotone, the following two conditions are equivalent:

(BRI) $G$ generates a positive $C_{0}$-semigroup $\left\{S_{t}: t \geq 0\right\}$ satisfying $\left\|S_{t}\right\|_{+} \leq 1$;

(BR2) $G$ is densely defined, $N$-dissipative and $R(1+\alpha G)=B$ for some $\alpha>0$.

Since the norms of $B$ and $B^{*}$ are monotone when $B$ is orthogonally decomposable, this theorem is applicable to our case. We recall that $G$ is $N$-dissipative if and only if $(f, G a) \geq 0$ whenever $a \in D(G)$ and $f \in H(a)$. This is equivalent to that $N((1+a G) a) \geq N(a)$ for all $a \in D(G)$ and $\alpha>0$. (See [2], Theorems 2.5 and 3.1, and [3], Theorem 2.1.1.)

(1) $\Rightarrow(2)$. Since each $S_{t}$ is a transition map, we have $\left\|S_{t}\right\|_{+}=1$. Therefore $G$ generates a $C_{0}$-semigroup $\left\{S_{t}: t \geq 0\right\}$ with $\left\|S_{t}\right\|_{+}=1$ and $-G$ generates a $C_{0}$-semigroup $\left\{S_{-t}: t \geq 0\right\}$ with $\left\|S_{-t}\right\|_{+}=1$. Thus $G$ 
and $-G$ satisfy condition (BRI), and, hence, (BR2). This implies condition (2).

$(2) \Rightarrow(1)$. It follows from the assumption that $G$ and $-G$ satisfy condition (BR2) and, hence, they generate $C_{0}$-semigroups $\left\{S_{t}\right\}$ and $\left\{T_{t}\right\}$ satisfying $\left\|S_{t}\right\|_{+} \leq 1$ and $\left\|T_{t}\right\|_{+} \leq 1$. However, since $T_{t}=S_{t}^{-1}, S_{t}$ are bi,jections and

$$
\begin{aligned}
N\left(S_{t} a\right) & =\sup \left\{\left(f, S_{t} a\right): f \in B_{+}^{*},\|f\| \leq 1\right\} \\
& \leq\left\|S^{*} f\right\| N(a) \leq N(a)
\end{aligned}
$$

for every $a \in B$, because $\left\|S_{t}^{*}\right\|_{+}=\left\|S_{t}\right\|_{+}$when $B$ is orthogonally decomposable. Similarly, we have $N\left(S_{t}^{-1} a\right) \leq N(a)$ for every $a \in B$. Thus $\left\{S_{t}\right\}$ are automorphisms by (4.I).

\section{References}

[1] T.Ando, "Majorization, doubly stochastic matrices and comparison of eigenvalues" (Research Institute of Applied Electricity, Hokkaido University, Sapporo, Japan, 1982).

[2] Wolfgang Arendt, Paul R. Chernoff and Tosio Kato, "A generalization of dissipativity and positive semigroups", J. Operator Theory 8 (1982), 167-180.

[3] Charles J.K. Batty and Derek W. Robinson, "Positive one-parameter semigroups on ordered Banach spaces" (Research Report, 5. Mathematical Science Research Centre, Australian National University, Canberra, 1983).

[4] Ola Bratteli and Derek W. Robinson, Operator algebras and quantion statistical mechanics I (Springer-Verlag, Berlin, Heidelberg, New York, 1979).

[5] Bruce Donald Calvert, "Nonlinear equations of evolution and fixed points" (PhD Dissertation, University of Chicago, Chicago, 1969). 
[6] Joseph Diestel, Geometry of Banach spaces - selected topics (Lecture Notes in Mathematics, 485. Springer-Verlag, Berlin, Heidelberg, New York, 1975).

[7] S. Kakutani, "Concrete representations of abstract ( $L$ ) spaces and the mean ergodic theorem", Ann. of Math. 42 (1941), 523-537.

[8] Dan Pascali and Silviu Sburlan, Nonlinear mappings of monotone type (Sijthoff and Noordhoff, Alphen aan den Rijn, 1978).

[9] J.J. Moreau, "Fonctionelles convexes" (Séminaire sur les équations aux derivées partielles, Collège de France, , 1966-1967).

[10] Derek W. Robinson, "Continuous semigroups on ordered Banach spaces", J. Funct. AnaZ. 51 (1983), 268-284.

[11] Derek W. Robinson and Sadayuki Yamamuro, "The canonical half-norms, dual half-norms and monotonic norms", Tôhoku Math. J. (2) 35 (1983), 375-386.

[12] Derek $W$. Robinson and Sadayuki Yamamuro, "The Jordon decompositions and half-norms", Pacific J. Math. 110 (1984), 345-353.

[13] Sadayuki Yamamuro, "On linear operators on ordered Banach spaces", Bull. Austral. Math. Soc. 27 (1983), 285-305.

[14] Sadayuki Yamamuro, "Half-norms and positive functions on ordered Banach spaces" (Research Report, 18. Department of Mathematics, Institute of Advanced Studies, Australian National University, Canberra, 1983).

Department of Mathematics, Institute of Advanced Studies, Australian National University, GPO Box 4, Canberra, ACT 2601 . Australia. 